\title{
SCALE-ADAPTIVE FILTERS FOR THE DETECTION/SEPARATION OF COMPACT SOURCES
}

\author{
D. Herranz ${ }^{1,2}$, J.L. Sanz ${ }^{1}$, R.B. Barreiro ${ }^{3}$ and E. Martínez-González ${ }^{1}$ \\ (1) Instituto de Física de Cantabria, Fac. de Ciencias, Av. de los Castros s/n, \\ 39005-Santander, Spain \\ (2) Departamento de Física Moderna, Universidad de Cantabria, 39005-Santander, Spain \\ (3) Astrophysics Group, Cavendish Laboratory, Madingley Road, Cambridge CB3 0HE, UK
}

Received _ ; accepted _ 


\begin{abstract}
This paper presents scale-adaptive filters that optimize the detection/separation of compact sources on a background. We assume that the sources have a multiquadric profile, i. e. $\tau(x)=\left[1+\left(x / r_{c}\right)^{2}\right]^{-\lambda}, \lambda \geq \frac{1}{2}, x \equiv|\vec{x}|$, and a background modeled by a homogeneous and isotropic random field, characterized by a power spectrum $P(q) \propto q^{-\gamma}, \gamma \geq 0, q \equiv|\vec{q}|$. We make an n-dimensional treatment but consider two interesting astrophysical applications related to clusters of galaxies (Sunyaev-Zel'dovich effect and X-ray emission).
\end{abstract}

Subject headings: methods: analytical — methods: data analysis — techniques: image processing

\title{
1. Introduction
}

The detection of localized signals or features on one-dimensional (1D) spectra or two-dimensional (2D) images is one of the most challenging aspects of image analysis. Astronomical spectra and images, for example, contain information that comes from a wide variety of physical phenomena mixed with instrumental noises of diverse origin (white noise, $1 / f$ noise, etc.). Before the analysis of such images it is necessary to carefully perform a component separation in order to isolate the different physical sources that contribute to the data. In this work we are interested in optimizing the detection of localized sources with spherical symmetry and spatial profiles belonging to a given family of mathematical functions. In particular, we will consider the well-known $\beta$ profiles of galaxy clusters that are broadly used in X-ray and microwave Astronomy. By 'detection' we mean the determination of the position of the sources as well as the estimation of parameters such as the intensity at the central pixel (amplitude, hereafter) and the characteristic scale of 
each source. Given the radial profile of the cluster, knowing the amplitude and the scale is equivalent to knowing the total flux at the observation frequency. Therefore, the whole 'detection' problem includes two different challenges: on the one hand, how to maximize the probability of finding a source against the noisy background in which it is embedded; on the other hand, how to accurately estimate the desired source parameters. Another related problem is how to distinguish between 'true' detections (i.e. those which correspond with real objects in the data) and spurious ones.

Given several images at different frequency channels, it is possible to use the knowledge about the frequency dependence of the different components as well as their statistical properties to separate them. Wiener filtering (WF, Tegmark and Efstathiou 1996; Bouchet et al. 1999) and Maximum Entropy Method (MEM, Hobson et al. 1998, 1999) are powerful tools for component separation based on this idea. When the frequency dependence of the sources is not known the previous methods are inefficient. Moreover, if only a single image is provided, the knowledge of the $\nu$-dependence of the different components is of no use. In that case, only spatial properties (such as characteristic scale, profile, structure, distribution and other statistical properties) might be used to perform the separation. In the case we are going to consider in this work, we intend to detect localized sources (that is, with a 'small' characteristic scale) with spherical symmetry and a given radial profile. All the other components in the image will be considered as a background ('noise') that should be removed in order to optimize the detection of the signal.

The classical treatment to remove the background has been filtering. A classical, maybe somewhat engineer-oriented definition of filter is: 'a filter is a device in the form of a piece of physical hardware or computer software that is applied to a set of noisy data in order to extract information about a prescribed quantity of interest' (Haykin 1996). This reflects the intuitive idea of a filter: a tool that, provided a certain data input, gives an 
output that have some desirable properties. From the signal processing point of view, a filter is a kind of system, that is, a process that results in the transformation of a signal. From the mathematical point of view, a filter is an operator:

$$
L: f(\vec{x}) \longrightarrow g(\vec{x})=L f(\vec{x})
$$

where $f$ is the input, $g$ is the output and $\vec{x}$ is the n-dimensional independent variable. Here we are interested in operators (filters) that are linear and that have translational invariance, that is, that if the input is translated by $\vec{p}$ the output is also translated by $\vec{p}$, that is, $g(\vec{x}-\vec{p})=L f(\vec{x}-\vec{p})$. A well-know result of signal processing theory is that any linear operator (filter) with translational invariance is equivalent to a convolution of the data with a certain function(convolution property). Any convolution can be expressed as a product in Fourier space. Thus, linear operators (filters) with translational invariance are frequency-selective (in the Fourier sense) devices: a filter selects or removes some of the features of the image depending on their frequency or, if the independent variable has dimensions of length, their scale. For example, Gaussian filters have been thoroughly used to suppress white noise from astronomical images. Low and high-pass filters are efficient in removing the small and the large features of an image, respectively, but are extremely inefficient in dealing with localized sources since many waves are required to represent such sources in Fourier space.

Wavelet formalism is well suited to deal with localized signals. The localized bases used in wavelet analysis allow one to obtain a precise representation of local objects both in spatial and frequency domains. In the context of signal processing, wavelets can be considered as a subset of the set of band-pass filters, capable of selecting a finite range of frequencies (scales) of an image, with the particularity that they are characterized not only by a translation but also by a scaling. This scaling allows one to perform a multiresolution analysis. The Mexican Hat wavelet (MHW) has been successfully applied to real and 
simulated X-ray images (Grebenev et al. 1995, Damiani et al. 1997, Valtchanov et al. 2001, Freeman et al. 2001) in order to detect X-ray sources, as well as to detect and extract point sources from simulated microwave maps (Cayón et al. 2000, Vielva et al. 2001a, 2001b). Another kind of wavelet that has been used in X-Ray Astronomy is the difference of two Gaussians (Rosati et al. 1995, Vikhlinin et al. 1998, Lazzati et al 1999).

We may wonder if a given wavelet such as the Mexican Hat or the difference of two Gaussians is the best possible choice in every case or if, on the contrary, there are different families of functionals (operators), wavelets or not, which are better suited to each particular case. It is clear that such an operator (filter) should take into account the shape (profile) of the source, its characteristic scale and the statistical properties of the background in which is embedded. In this context, the application of matched filtering (MF) was recognized to be an effective method for detection of faint sources (see for example Irwin 1985). MF has been applied to the detection of faint sources in ROSAT PSPC images (Vikhlinin 1995). Regarding CMB data analysis, Tegmark \& Oliveira-Costa (1998) introduced a matched filter that minimizes the variance of the filtered map. Their filter takes maximum advantage of the knowledge of the source profile and the background properties, but it does not explicitly require that the detection has to be optimal at a fixed scale, i.e., it is not optimal to detect sources of a given scale and only them. On the other hand, Sanz et al. (2001) introduced a family of filters (pseudofilters or scale-adaptive filters) that produce maximum detection at a particular scale. Optimal scale-adaptive filters have been successfully used to detect and extract point sources from simulated microwave time ordered data sets (Herranz et al. 2002).

Sanz et al. (2001) showed that the MHW is nearly optimal to detect point sources convolved with a Gaussian beam because of the relation between the MHW and the laplacian of the Gaussian. If the source profile is other than Gaussian, however, the optimal 
filter would be different. For example, an astronomical image may contain different kind of objects with different spatial profiles. In that case, it would be necessary to use different filters to detect each kind of objects. In this work we will apply optimal scale-adaptive filters to the separation and detection of compact sources with a multiquadric profile. Such profile is quite common in X-Ray and CMB images. In section 2 we briefly describe the formalism of scale-adaptive filters and introduce the multiquadric profiles that we are going to study as well as the concept of 'gain'. Section 3 introduces other related filters such as the MHW and the matched filter. A more extensive discussion about the differences between scale-adaptive filters and wavelets is presented in this section. In section 4 the scale-adaptive filters are applied to simple simulations of multiquadric profiles embedded in homogeneous and isotropic backgrounds. Section 5 deals with an empirical comparation between the scale-adaptive filters and two examples of wavelets, namely the Mexican Hat Wavelet and the difference of two Gaussians. Finally, in section 6 we discuss our conclusions.

\section{The scale-adaptive filter}

\subsection{The concept of scale-adaptive filter}

Let us consider an n-dimensional (nD) image with data values defined by

$$
y(\vec{x})=s(x)+n(\vec{x}), \quad x \equiv|\vec{x}|,
$$

where $\vec{x}$ is the spatial coordinate and $s(x)$ represents a compact source with spherical symmetry and a characteristic scale (e.g. a single maximum at its center and decay at large distances) placed at the origin. The background $n(\vec{x})$ is modeled by a homogeneous and isotropic random field with average value $\langle n(\vec{x})\rangle=0$ and power spectrum $P(q), q \equiv|\vec{q}|$ :

$\left\langle n(\vec{q}) n^{*}\left(\overrightarrow{q^{\prime}}\right)\right\rangle=P(q) \delta_{D}^{n}\left(\vec{q}-\overrightarrow{q^{\prime}}\right), n(\vec{q})$ is the Fourier transform of $n(\vec{x})$ and $\delta_{D}^{n}$ is the nD Dirac distribution. 
The idea of an optimal pseudo-filter (or a scale-adaptive filter) has been recently introduced by the authors (Sanz et al. 2001). Let us consider a generic sphericallysymmetric filter, $\Psi(\vec{x} ; R, \vec{b})$, dependent on $n+1$ parameters ( $R$ defines a scaling whereas $\vec{b}$ defines a translation)

$$
\Psi(\vec{x} ; R, \vec{b})=\frac{1}{R^{n}} \psi\left(\frac{|\vec{x}-\vec{b}|}{R}\right) .
$$

This generic filter is very similar to a continuous wavelet transform, i.e. it is characterized by self-similarity (or covariance under $\vec{x} \rightarrow R(\vec{x}-\vec{b})$ transformation). In fact, $\Psi$ may be a wavelet if the wavelet transform conditions (see section 3.1) are met. Let us consider not only wavelets but the whole set of filters that can be described by eq. (3). We define the filtered field as

$$
w(R, \vec{b})=\int d \vec{x} y(\vec{x}) \Psi(\vec{x} ; R, \vec{b})
$$

Now, we are going to express the conditions in order to obtain an optimal (in the sense that will be described below) scale-adaptive filter for the detection of the source $s(x)$ at the origin taking into account the fact that the source is characterized by a single scale $R_{o}$. By 'optimal' we mean that the filter must satisfy the following conditions: (i) $\left\langle w\left(R_{o}, \overrightarrow{0}\right)\right\rangle=s(0) \equiv A$, i. e. $w\left(R_{o}, \overrightarrow{0}\right)$ is an unbiased estimator of the amplitude of the source, (ii) the variance of $w(R, \vec{b})$ has a minimum at the scale $R_{o}$, i. e. it is an efficient estimator of the amplitude of the source, and (iii) $w(R, \vec{b})$ has a maximum at $\left(R_{o}, \overrightarrow{0}\right)$. The meaning of these conditions is simple: in order to maximize the probability of detection one wants to reduce as far as possible the contribution of noise (condition (ii)) while preserving the sources (condition (i)). Additionally, condition (i) provides a normalization that gives directly the amplitude of the sources after filtering. Conditions (i) and (ii) alone give birth to the so-called matched filter. It is a well-known result of signal analysis that matched filter produces the maximum gain in SNR when going from real to filter space. By introducing further conditions in the making of the filter we constrain the space of functionals from 
which the filters are drawn, thus reducing the final SNR gain of the filter. Nevertheless, we introduce a third condition in our definition of the 'optimal filter'. This last condition is set in order to optimize the characterization of the scale of the source by the filter. As we will see in further sections, condition (iii) allows one to establish a straightforward relation between the filter parameters and the characteristic scale of the sources to be detected. Besides, it helps to reduce the probability of false detections. A filter satisfying conditions (i) to (iii) is optimal for finding the sources, determining their scales and amplitudes and discarding spurious detections at the same time.

The filter satisfying conditions (i), (ii) and (iii) is given by the equation

$$
\begin{aligned}
\tilde{\psi}(q) \equiv \psi\left(R_{o} q\right) & =\frac{1}{\alpha \Delta} \frac{\tau(q)}{P(q)}\left[n b+c-(n a+b) \frac{d l n \tau}{d l n q}\right], \\
\Delta & \equiv a c-b^{2}, \quad \alpha \equiv \frac{2 \pi^{n / 2}}{\Gamma\left(\frac{n}{2}\right)}, \\
a \equiv \int d q q^{n-1} \frac{\tau^{2}}{P}, \quad b & \equiv \int d q q^{n} \frac{\tau}{P} \frac{d \tau}{d q}, \quad c \equiv \int d q q^{n+1} \frac{1}{P}\left(\frac{d \tau}{d q}\right)^{2} .
\end{aligned}
$$

where $\tau$ is the profile of the source in Fourier space $(s(q)=A \tau(q))$. Generically, $\Psi$ is not positive. Moreover, as mentioned above $\Psi$ does not define in general a continuous wavelet transform, although it has its self-similarity property. We could obtain a continuous wavelet transform in the same way described above simply by introducing in the derivation of the filter the additional conditions that a wavelet transform must satisfy. However, this would lower the SNR gain. This would reduce the usefulness of the filter from the point of view of pure detection. Therefore, we will not introduce any other condition.

The filter given by eq. (5) adapts to the source profile, the background and the scale of the source, i. e. the name scale - adaptive filter. Taking into account equation (4), the amplitude is estimated as

$$
A=w\left(R_{o}, \overrightarrow{0}\right)=\int d \vec{q} y(\vec{q}) \tilde{\psi}(q),
$$


whereas an estimation of the error is given by the dispersion $\sigma_{w}$ of the filtered image

$$
\sigma_{w}=\left[\left\langle\left(w\left(R_{o}, \vec{b}\right)\right)^{2}\right\rangle-A^{2}\right]^{1 / 2}
$$

with the average including all points $\vec{b}$ in the image. This equation provides a theoretical estimation of the variance of the estimation of the amplitude of sources.

The previous equations have been used by the authors to obtain the scale-adaptive filter for a Gaussian and an exponential profile (Sanz et al. 2001).

\subsection{Multiquadric profile}

In some astrophysical/cosmological applications the source is modeled by a multiquadric, i. e. the profile is given by

$$
\tau(x)=\frac{1}{\left[1+\left(\frac{x}{r_{c}}\right)^{2}\right]^{\lambda}}, \quad \lambda \geq 1 / 2 .
$$

Typical examples are, for the 2D case, the emissions in the microwave and X-ray bands with $\lambda=\frac{3 \beta-1}{2}, \frac{6 \beta-1}{2}$, respectively, for a $\beta$-profile for the electron number density

$n_{e}(r) \propto\left[1+\left(x / r_{c}\right)^{2}\right]^{-\frac{3}{2} \beta}$. Assuming the standard value $\beta=2 / 3$ one trivially obtains $\lambda=1 / 2,3 / 2$ for the microwave and X-ray emissions, respectively.

Assuming a scale-free power spectrum $P(q)=D q^{-\gamma}$, the equations (5-7) lead to the filter

$$
\begin{gathered}
\tilde{\psi}(q)=\frac{1}{\alpha a^{\prime}} \frac{\Gamma(\lambda)}{2^{1-\lambda}(\gamma+n)}\left(q r_{c}\right)^{\gamma+\lambda-\frac{n}{2}}\left[P K_{\lambda-\frac{n}{2}}\left(q r_{c}\right)+Q q r_{c} K_{1+\lambda-\frac{n}{2}}\left(q r_{c}\right)\right], \\
P \equiv 2 \gamma-(n-\gamma)(\gamma+2 \lambda), \quad Q \equiv 2(n-\gamma) \frac{\gamma+2 \lambda+1}{\gamma+4 \lambda-n} \\
a^{\prime} \equiv \frac{2^{\gamma+2 \lambda-3}}{\Gamma(\gamma+2 \lambda)} \Gamma\left(\frac{\gamma+n}{2}\right) \Gamma^{2}\left(\frac{\gamma+2 \lambda}{2}\right) \Gamma\left(\frac{\gamma+4 \lambda-n}{2}\right) .
\end{gathered}
$$


In the previous equations $\Gamma$ denotes the Gamma function whereas $K$ denotes the Bessel $K$ function. Table 1 gives the analytical form of the scale-adaptive filter for $\lambda=1 / 2,3 / 2$ and different values of the spectral index $\gamma=0,1,2,3$ on Fourier space and real space. The 2D scale-adaptive filters for the same parameters are shown in the left side of figure 1.

\subsection{Detection level and gain}

One can define the detection level on real $(D)$ and filtered $\left(D_{w}\right)$ space as

$$
\mathcal{D}=\frac{A}{\sigma}, \quad \mathcal{D}_{w}=\frac{A}{\sigma_{w}}, \quad g=\frac{\sigma}{\sigma_{w}}
$$

where $\sigma, \sigma_{w}$ are the dispersion in the real image and filtered image, respectively. The gain $g$ gives the amplification of the sources achieved in filter space with respect to the original image.

The most straightforward way to identify sources in an image is to look for peaks of the signal above a certain threshold. In Astronomy is usual to use thresholds that are a certain number of times the dispersion of the image. Therefore, the number of faint sources that can be detected with a filter is proportional to the gain of the filter. As mentioned above, conditions (i) and (ii) alone (corresponding to a matched filter) guarantee the maximum possible gain; if additional conditions are required the gain will be lower.

Other issue that has to be taken into account is the reliability of the detection. The identification of sources as peaks above a certain threshold (e. g. $3 \sigma_{w}$ ) in real or in filter space gives a finite probability of false detections due to noise fluctuations. If the noise is Gaussian and uncorrelated it is easy to control the false discovery rate simply by choosing the corresponding $\sigma$ level threshold. Unfortunately, this is not true in many cases. In general, the relation between the value of the threshold and the false discovery rate is not straightforward and must be calibrated in some way, for example using simulations. 
A compromise between sensitivity and reliability must be reached. A way to overcome this problem is to use additional information about the sources in order to distinguish them from the noise fluctuations. In many cases, one looks for objects that have a fixed scale in the image. As an example, point sources in CMB maps appear as Gaussian-like features (due to the beam psf) with the scale of the beam width. Scale-adaptive filters are specifically designed to take advantage of the characteristic scale of the sources in order to reduce spurious detections. By means of condition (iii), only features that have the same characteristic scale of variation as the sources we intend to detect are enhanced. In other words, the gain experimented by a background fluctuation drops quickly when it has a characteristic scale of variation different from that of the sources.

\section{Other filters}

For comparison with the filters developed in the previous section, we shall briefly introduce other filters that have been extensively used in the literature. Two of them, the Mexican Hat and the difference of two Gaussians, are wavelets. The other is the well-known 'matched' filter (MF).

\subsection{Wavelets and filters}

The development of wavelet techniques applied to signal processing has been very fast in the last ten years. A wavelet is a 'small wave' which has its energy concentrated in space and allows simultaneous space and scale (frequency) analysis based on location and similarity. Wavelets are so useful because of their good space-frequency localization. Besides, almost all useful wavelet systems allow one to reconstruct a given signal in terms

of a wavelet basis that is generated from a single scaling function by simple scaling and 
translation. Eq. (3) shows these two basic operations. There is not a unique choice for the wavelet basis. The ability of giving better approximations to a signal by means of successive 'refinements' of the wavelet is called the multiresolution condition. In order to have a wavelet (i.e. to allow a multiresolution analysis and the reconstruction of signals), the functions in eq. (3) must satisfy a number of additional conditions, namely the condition $\int d \vec{x} \Psi=0$ and the 'admissibility' condition $\int d q q^{-1} \psi^{2}(q)<\infty$. Due to their good space-frequency localization and their ability to perform a complete decomposition of the signal, wavelets are ideal tools for identifying features in images, denoising and data compression.

From de point of view of mere source detection, however, not all the properties of wavelets are equally necessary. Clearly, good space-frequency localization is an important condition to be met. On the contrary, a full decomposition and posterior reconstruction of the image is not necessary, since we are only interested in finding the position of the sources and a handful of parameters such as the amplitude; as explained in the previous section, each new condition to be satisfied by a filter reduces the SNR gain in the output. In this context, the filter (5) resembles a wavelet in the sense of space-frequency localization, but is more generic and therefore it will produce higher gains (and, therefore, will be more sensitive to weak sources).

A different approach to the detection problem is the denoising of images previous to the actual detection. Since noise usually manifests at scales different from the source characteristic scale, scale-adaptive filters are good at denoising. Wavelet-based denoising algorithms are also very powerful and efficient (as an example applied to CMB data analysis see Sanz et al. 1999a,b). Wavelet-oriented denoising techniques are out of the scope of this work (for an introduction to this topic see for example Odgen 1997). 


\subsubsection{The Mexican Hat wavelet}

The well-known Mexican Hat wavelet is defined by

$$
\begin{gathered}
\Psi(\vec{x} ; R, \vec{b})=\frac{1}{R^{n}} \psi\left(\frac{|\vec{x}-\vec{b}|}{R}\right), \quad \psi(x) \propto\left(n-x^{2}\right) e^{-x^{2} / 2} \\
\psi(q) \propto q^{2} e^{-\frac{1}{2} q^{2}}
\end{gathered}
$$

This type of wavelet has been extensively used for point source detection. Optical images of galaxy fields have been analyzed to detect voids and high-density structures in the first CfA redshift survey slice (Slezak et al. 1993). Microwave images have been analyzed (Cayón et al. 2000; Vielva et al. 2001a) and combined with the maximum entropy method (Vielva et al. 2001b) to obtain catalogs of point sources from simulated maps at different frequencies that will be observed by the future Planck mission. On the other hand, the MHW has also been used to detect X-ray sources (Grebenev et al. 1995, Damiani et al. 1997) and presently for the on-going XMM-Newton mission (Valtchanov et al. 2001) and Chandra (Freeman et al. 2001).

The Mexican Hat Wavelet is well suited to deal with Gaussian structures due to its relation with the Laplacian of the Gaussian. Intuitively, it gives good results because its profile is highly correlated with the Gaussian profile. In fact, Sanz et al. 2001 showed that the scale-adaptive filter for a two-dimensional Gaussian source embedded in a noise characterized by the power spectrum $P(q) \propto q^{-\gamma}$ with $\gamma=0$ (white noise) and $\gamma=2$ coincides with a Mexican Hat Wavelet. If the source profile is not Gaussian or the background is not described by the relation above, the MHW is not optimal. 


\subsubsection{Difference of two Gaussians}

A wavelet kernel can be constructed by subtracting two Gaussians. For example, in the two-dimensional case

$$
\Psi(\vec{x} ; R, \vec{b})=\frac{1}{R^{2}} \psi\left(\frac{|\vec{x}-\vec{b}|}{R}\right), \quad \psi(x)=2\left[e^{-x^{2}}-\frac{1}{2} e^{x^{2} / 2}\right] .
$$

This wavelet shows a positive core and a outer negative ring, and the convolution with any linear function $s(x, y)=a x+b y+c$ is zero (this is also true for the Mexican Hat). Therefore, any slowly varying background that can be locally approximated by such kind of linear functions is subtracted by this wavelet. The difference of two Gaussians has been applied to detect X-ray sources (Rosati et al. 1995, Vikhlinin et al. 1998, Lazzati et al 1999).

\subsection{The matched filter}

If one removes condition (iii) defining the scale - adaptive filter in the previous section, another type of filter can be found after minimization of the variance (condition(ii)) with the constraint (i)

$$
\tilde{\psi}_{m}(q)=\frac{1}{\alpha a} \frac{\tau(q)}{P(q)}
$$

This is usually called a matched filter. In general, the matched and scale - adaptive filters

are different. In the former case, one obtains a slightly larger gain (although for $\gamma \geq 0.5$ the gain is the same from the practical point of view, i.e. the relative difference is less than 20\%) but sources must be identified 'a posteriori' with an extra criterion whereas the scale - adaptive filter allows one to get the sources in a straightforward manner. Thus, from the methodological point of view and reliability (detection of spurious sources) it is better to use the scale - adaptive filter unless the image is completely dominated by white noise. 
Matched filters have been used recently to detect clusters of galaxies from optical imaging data (Postman et al. 1996; Kawasaki et al. 1998) and sources from X-ray images (Vikhlinin 1995). In this approach the method uses galaxy positions, magnitudes and photometric/spectroscopic redshifts if available to find clusters and determine their redshift.

For the case of a source profile given by eq. (10) and a scale-free power spectrum given by $P(q) \propto q^{-\gamma}$, the previous formula (18) leads to the following matched filter

$$
\tilde{\psi}_{m}(q)=\frac{1}{\alpha a^{\prime}} \frac{\Gamma(\lambda)}{2^{1-\lambda}}\left(q r_{c}\right)^{\gamma+\lambda-\frac{n}{2}} K_{\lambda-\frac{n}{2}}\left(q r_{c}\right),
$$

with $a^{\prime}$ given by eq. (13). For $\lambda=1 / 2$ the matched filter is not defined for white noise (i.e. $\gamma=0$ ), whereas for other values of $\gamma$ is given by table 2 in Fourier space and real space, respectively. The same filters appear in the right side of figure 1. Note that the scale-adaptive filters reach their peak around $q r_{c} \sim 1$, whereas there is more dispersion in the position of the peak of the matched filters. This means that scale-adaptive filters enhances the signal against the background in q-space at scales comparable with the characteristic scale of the source. We remark that for $\gamma=n$ the scale-adaptive filter and the matched filter coincide. For the $\lambda=1 / 2$ and $\gamma=0$ case, we have assumed the modified profile

$$
\tau(x)=N\left[\frac{1}{\left(r_{c}^{2}+x^{2}\right)^{1 / 2}}-\frac{1}{\left(r_{v}^{2}+x^{2}\right)^{1 / 2}}\right], \quad N \equiv \frac{r_{c} r_{v}}{r_{v}-r_{c}},
$$

where $r_{v}$ is a cut-off radius. The behavior of this profile is: $\tau(0)=1$ and $\tau(x) \propto x^{-3}$ for $x \gg r_{v}$.

\section{Numerical simulations: application and results}

In order to show the performance of the scale-adaptive filters we have simulated realizations of multiquadric profiles embedded in backgrounds of the type $P(q)=D q^{-\gamma}$. 
For the sake of simplicity we simulate ideal multiquadric profiles, not taking into account the effect of the detector beam. However, the method can be generalized to include this effect by modifying the input source profile. We also consider only integer values of $\gamma$, so we can directly use the filters from Table 1 . In a more realistic case, where the power spectrum does not follow such a simple law, it could be directly estimated from the data and the filter numerically calculated.

\subsection{Microwave emission and the SZ-effect of clusters}

\subsubsection{SZ clusters of equal size}

One of the most promising applications of scale-adaptive filters is the detection and extraction of the emission of galaxy clusters due to the Sunyaev-Zel'dovich (SZ) effect in microwave maps. Maps of the Cosmic Microwave Background (CMB) contain contributions

from a variety of foregrounds (the SZ effect among them) and different types of noise. If we approximate the power spectrum of a typical CMB map by a $P(q)=D q^{-\gamma}$ law, the effective index $\gamma$ ranges between values near 0 (for regions dominated by white noise) to $\sim 3$ (for regions dominated by dust emission). As an example, we first simulated a $512 \times 512$ pixel field containing 100 randomly distributed 'clusters' with a multiquadric profile $(\lambda=1 / 2)$. All the 'clusters' have the same scale $\left(r_{c}=1.0\right.$ pixel $)$ and amplitudes distributed between 0.1 and 1 (in arbitrary units). With a convenient rescaling of the amplitude, this could simulate, for example, a $12^{\circ} .8 \times 12^{\circ} .8$ field of the sky filled with clusters of several arcmin of extent. The simulated clusters are shown in the left panel of figure 2. A $P(q)=D q^{-3}$ background was added so that the peaks of sources are on average at the $2 \sigma$ level of the final map. The map containing the sources and the background ('noise') is shown in the center of figure 2 . In the following, we will call this simulation 'simulation 1'. 
Following table 1, the scale-adaptive filter for the case $\lambda=1 / 2, \gamma=3$ is $\tilde{\Psi}_{o}(q)=4(3-y) y^{2} e^{-y} / 3 \pi, y=q r_{c}$. The result of applying the scale-adaptive filter on the simulated field is shown in the right panel of figure 2. For the sake of clarity, only the pixels above the $3 \sigma$ level have been plotted. After filtering, the detection is performed by looking for peaks above a certain threshold. Knowing the initial position and amplitude of the simulated sources, it is possible to determine quantities such as the mean error in the estimation of the source parameters as well as the reliability of the filter (that is, the probability of detecting spurious 'sources'). The results are summarized in table 3 . The first column in table 3 shows the $\sigma$ threshold used for the detection. The second column shows the number of true detections found. The third column indicates the number of spurious 'sources' detected. The fact that above $4 \sigma$ we find more spurious detections than above the $3 \sigma$ threshold may seem surprising. This can be easily explained taking into account that our detection method looks for sets of connected pixels over a given threshold. Therefore, a 'lump' with two peaks may be seen as a single source if the detection threshold is low enough whereas a higher detection threshold may split the 'lump' into separated peaks. The fourth column in table 3 indicates the mean error in the determination of the position of the source in pixel units. As can be seen, all the detected sources were correctly located. The mean relative bias given in the fifth column of table 3 is defined as $\bar{b}_{A}(\%)=100 \times \frac{1}{N} \sum\left(A_{i}-A_{o_{i}}\right) / A_{o_{i}}$, where $A_{o_{i}}$ and $A_{i}$ are the original and estimated amplitudes of the sources respectively and $N$ is the number of considered sources. The mean relative error given in the sixth column is defined as $\bar{e}_{A}=100 \times \frac{1}{N} \sum\left|A_{i}-A_{o_{i}}\right| / A_{o_{i}}$. The seventh column gives the mean gain, as defined in eq. (13). The most striking fact in this case is the high gain $(\sim 4.4)$ that is found in all the cases. This is due to the fact that the scale-adaptive filter (which acts as a band-pass filter) is very efficient in removing the large scale structure that characterizes the $q^{-3}$ background. The individual gain for each detection as a function of the true amplitude of the source is shown in the bottom panel of 
figure 3. We see that there is a certain dispersion around the average value (represented in figure 3 as an horizontal dotted line) but, in general, the gain is nearly independent of the original amplitude of the source. Only for the brightest and the faintest sources this independence is lost. Bright sources tend to have lower gains than the average and vice versa. This is related to what is seen in the top panel of figure 3, which shows the estimated amplitudes of the detected sources versus the original amplitudes. Between the original and estimated amplitudes there is a strong linear regression but with a small positive deviation from the $y=x$ law. This corresponds to the $\sim 7 \%$ relative bias that is given in table 3 . Again, both bright and faint sources deviate from this law.

Figure 3 can be explained considering the detection strategy we use. We look for peaks above a certain threshold, that is, for local maxima in the filtered image. In an ideal case, the value of the peak gives directly the amplitude $A$ of the sources (due to condition (i) of scale-adaptive filters). Unfortunately, a certain amount of statistical residual noise is always found in the filtered images. This noise is superimposed to the signal peaks and produces an error in the estimation of $A$. This error can be positive or negative. Since our selection criterion involves looking for local maxima, positive errors are more likely to be detected than negative ones. This leads to a systematic statistical positive bias. This effect has more relevance for faint sources due to the fact that the relative contribution of the residual noise to the signal is higher in this case.

There is one more reason for the higher gain in the case of faint sources. The number counts of faint sources (near the detection level) is affected by the well-known 'detection bias'. Only the sources that are more amplified than the average can be detected; therefore, the mean gain of weak sources is higher than the mean gain of bright ones. From eqs. (8) and (9) is straightforward to show that the gain of the scale-adaptive filter does not depend on the amplitude of the source. Therefore, the differences among the gains obtained in 
different sources is caused by the fluctuations due to residual noise.

As $A_{o}$ increases in the top panel of figure 3 , the bias decreases and eventually drops to negative values. This suggests the existence of a systematic effect that gives a negative bias and that is compensated by the positive bias produced by the detection method when the sources are weak enough. Such negative bias has been reported in relation with scale-adaptive filters in Sanz et al 2001 and Herranz et al. 2002, and is due to two kinds of effects. On the one hand, the normalization given by condition (i) of scale-adaptive filters is calculated for an ideal profile on an infinite, continuous field (the limits of the integral are 0 and $\infty$ ), while the real images are finite and discrete (pixelized). On the other hand, the correlation between the (analytic) filter (5) and the discrete, pixelized, 'real' data is not perfect.

In spite of these small systematics, the performance of the scale-adaptive filter is very good in the sense that with a simple application of a filter and a thresholding detection scheme we are able to recover a very significant number of sources and estimate their amplitudes with errors not larger than $\sim 10 \%$. The reliability of the method is such that over a $3 \sigma$ threshold there are a $15 \%$ of spurious detections. A possibility to remove this small bias is to calibrate it as a function of the amplitude of the sources by means of additional simulations using the same background and artificial sources with known amplitudes.

\subsubsection{SZ clusters with different sizes}

In a more realistic case the scale $r_{c}$ of the sources will not be known a priori. For example, in a CMB map, clusters of different scales will be present, going from almost point-like sources to large structures that extend across several pixels. Under these 
conditions, it does not seem clear which one should be the scale of the scale-adaptive filter. Fortunately, condition (iii) of the optimal scale-adaptive filters implies that the coefficient at the position of the source and at the 'right' scale is a maximum. Therefore, the strategy to follow is to filter the image with a set of $N$ filters with different 'tentative' scales $r_{c_{i}}$, $i=1, \ldots, N$. For a given detection, the maximum value among the coefficients at the position of the source of the different filtered maps will correspond to the $r_{c_{i}}$ closer to the $r_{c}$ of the cluster. This method is similar to a multiresolution analysis. The discrete wavelet transform (DWT) allows one to perform a multiresolution analysis by varying the scale of the analyzing wavelet in logarithmic samples $\left(2^{j}, j=1,2, \ldots, n\right)$. Such approach has been successfully used in X-ray data analysis (see for example Vikhlinin et al. 1998 and references therein). This allows an optimal decomposition of the signal in frequency space. Our approach is more similar to a continuous wavelet transform (CWT), in which the scale varies in a continuous way. Since we can not calculate an infinite number of continuous scale variations, we sample the scale space as densely as needed until we reach the desired precision. Thus, the expected error in the determination of $r_{c}$ should be the size of the 'step' in the sampling scales. In fact, the estimation of this error is more complicated and depends on the relative sizes of the core radius and the pixel, the characteristic scale of the background fluctuations and the shape of the sources (for example the value of $\lambda$ will be relevant for this matter). We suggest to estimate the expected errors by means of simulations. As a first approximation, however, we will consider through this work that the expected error is simply the size of the 'step' in the sampling scales.

To test this point we performed a new simulation (simulation 2, hereafter) with 100 'clusters' with $r_{c}$ distributed between 0.5 and 2.0 pixels and amplitudes between 0.1 and 1.0 (in arbitrary units). The noise is similar to that of simulation 1. The simulated map was filtered with scale-adaptive filters with the parameter $r_{c}=0.25,0.50,0.75,1.00,1.25,1.50$, $1.75,2.00,2.25$ and 2.50 pixels. In figure 4 we plot the original clusters (left panel), the 
simulated map with noise (central panel) and the map filtered with the scale-adaptive filter $r_{c}=1.50$ (right panel). The sources are detected over each filtered map by selecting peaks above a certain threshold. If one of such peaks is detected at the same position on different filtered maps, it is unlikely that it corresponds to pixel-scale noise (although it could be a fluctuation of the noise at a scale of the order of the source scale). Looking for detections in several filtered maps will help to reduce the number of spurious sources in the output. For every detection that is present in several filtered maps we look for the maximum of the coefficients at the central position. In particular, we only consider sources that appear at least at 5 scales. This number was chosen because with 5 consecutive scales it is possible to 'cover' the pixel size in the scale space and because it gives a good compromise between the number of detections and the number of spurious detections, as will be seen through this section. The maximum gives both the scale of the source and its amplitude.

The results are shown in table 4 . The main difference between tables 4 and 3 is the lower gains that are obtained in simulation 2. This result is expected because the set of filters used only fit clusters with some specific values of $r_{c}$. For the rest of clusters the filters are only approximately optimal. Moreover, any error in the determination of $r_{c}$ will lead to a wrong determination of the amplitude. The top panel of figure 5 shows the estimated $r_{c}$ versus the original $r_{c_{o}}$ in simulation 2. There is a significant dispersion around the $r_{c}=r_{c_{o}}$ line (represented by a dotted line in the top panel of figure 5). As shown in table 4 , the mean error in the determination of $r_{c}$ is $\sim 0.15$ pixels, with a similar bias towards estimating higher values of $r_{c}$ than the real ones. If we increase the 'resolution' in $r_{c}$ by increasing the number of filters the error is not significantly reduced. This indicates that it is not possible to determine scale parameters with a much better resolution than the pixel scale. In spite of this limitation, the errors in the determination of the amplitude remain reasonable $(\sim 10 \%)$. This indicates that the estimated amplitude does not vary significantly with $r_{c}$ in the neighborhood of the real value of the core radius. Therefore, 
errors under the pixel scale in the estimation of $r_{c}$ have little effect in the determination of the amplitude. Lower panel of figure 5 shows the estimation of the amplitudes in simulation 2. As in simulation 1, a small positive bias is found. The gain shows the same behavior with respect to the amplitude of the sources as in simulation 1 (an asymptotic decrease of the gain with the amplitude of the clusters) and is almost independent of the size of the clusters. Small clusters tend to have slightly higher gains than large clusters. This could be due to the fact that small clusters are detected in maps filtered with small $r_{c}$ parameter and therefore in these maps the pixel-scale residuals are worse removed than in maps filtered with a large $r_{c}$. Maps filtered with small $r_{c}$ show more contribution from pixel-scale noise residuals and therefore the peak amplitude is more likely to be overestimated. The number of spurious detections drops to 0 due to the fact that we discard those 'candidates' that are not detected in at least 5 filtered maps. Indeed a similar constraint is imposed in the MHW method used by Vielva et al. (2001a and 2001b), who performed a fit at several scales in order to estimate the amplitude of point sources as well as to discard spurious detections. Unfortunately, this constraint also reduces the number of true detections. This is an example of a well-known and unavoidable problem in detection theory: the cost of reducing spurious detections is to reduce the number of true detections and, conversely, relaxing the selection criteria to include more true sources leads to an increase of spurious detections.

\subsection{X-ray emission and clusters}

Other straightforward application of scale-adaptive filters is in the field of X-ray astronomy. X-ray emission from galaxy clusters roughly follows a multiquadric profile with $\lambda=3 / 2$. Unfortunately, real X-ray images suffer from strongly non-isotropic point spread functions that distort cluster profiles to quite odd and asymmetric shapes. For this work, 
however, we will consider (as we did in the previous case of SZ emission) that instrumental effects have been somehow corrected and that we only have ideal clusters and noise. For simulation 3 we distributed 100 ideal multiquadric profiles with $\lambda=3 / 2, r_{c}$ between 2.0 and 4.5 pixels and amplitudes between 0.1 and 1.0 (in arbitrary units). The dominating background in X-ray images is Poissonian shot-noise that, when the exposure time is long enough, can be approximated by white noise. We added white noise $(\gamma=0)$ to our simulation so that, given the amplitudes of the clusters (in their arbitrary units), the final signal to noise level is roughly similar to the one of an XMM image of $95 \mathrm{Ks}$ of exposure time. The reference X-ray data for this simulation was an image of the Lockman Hole field adquired in the 2-7 KeV energy band with the pn detector of the XMM-Newton satellite (with a pixel size of $4^{\prime \prime} \times 4^{\prime \prime}$ ). The background map (mean value of $10^{-5} \mathrm{cts} / \mathrm{s} / \mathrm{pixel}$ ) was estimated with the esplinemap SAS v5.2 task. Simulation 3 was performed so that the largest cluster size (in pixel units) and the signal to noise were approximately equal to the conditions in the central region of the reference image. The final signal to noise ratio is greater than in simulations 1 and 2. Figure 6 shows the simulated clusters (left panel), the complete simulation with noise (central panel) and the coefficient map that corresponds to the simulation filtered with an scale-adaptive filter with $r_{c}=3.0$ pixels.

The detection and determination of the amplitude and the scale of the clusters were performed following the same steps than in simulation 2. The set of chosen 'core radii' was $r_{c}=1.2,1.3,1.5, \ldots, 5.1$ pixels $(N=40$ filters $)$. To consider a detection as a source it must be present in at least ten of the filtered maps (we chose this number for the same reasons that were explained in simulation 2). The results are shown in table 5. Simulation 3 has two qualitative differences with respect to simulations 1 and 2. First of all, the cluster profile drops much faster in the X-ray case than in the SZ case. Second, the simulated background is white noise instead of $\propto q^{-3}$. This is not the optimal situation since the gain achieved by the scale-adaptive filter is not very high in comparison with other filters when 
the background is dominated by white noise. In particular, the gains obtained in simulation 3 are only $\sim 2$ (table 5$)$. Moreover, the fast decline of the cluster profile makes them much more compact (that is, more point-like), making more difficult to distinguish them from noise fluctuations. In other words, comparatively to a microwave image of the same pixel scale and in which there are clusters of the same $r_{c}$, it is harder to estimate parameters such as $r_{c}$ because the signal is condensed in a few pixels. If, for example, $r_{c}$ was of the order or less than half a pixel, the cluster would be almost indistinguishable from a point source. This explains why, in spite of having the $r_{c}$ space quite densely sampled, the mean error in the determination of that parameter is almost of 0.5 pixels. There is no observed bias in the determination of $r_{c}$. Clusters in simulation 2 had smaller core radii than those in simulation 3, and therefore it was more likely to overestimate than to underestimate their size. In simulation 2, smaller values of $r_{c}$ were chosen in order to avoid overlapping among the clusters (the compactness of X-ray clusters reduces the probability of overlapping). Besides, the $P(q) \propto q^{-3}$ noise fluctuations grow stronger with their scale, so large-scale residuals (that, when combined with the filtered profile, would lead to overestimation of the scale) were more likely in simulation 2 that pixel-scale residuals (that, when combined with the filtered profile, would lead to underestimation of the scale). Under white noise conditions all scales of the background have the same power and therefore there is no reason for bias in any of the two directions, as is observed.

Figure 7 shows the performance of scale-adaptive filters in the determination of the amplitude (bottom panel) and $r_{c}$ (top panel). The dispersion in the estimated $r_{c}$ is comparatively much greater than the dispersion in the estimated $A$ (lower panel of figure 7 ). The dispersion in the estimation of the amplitude is comparatively greater than in previous simulations due to the fact that the dispersion of the filtered map (that is, the intrinsic statistical error in the determination of the amplitude) is greater in simulation 3 than in simulation 1 and 2 . These errors, however, remain relatively small $(\sim 15 \%$, table 5$)$. The 
small relative errors in the determination of $A$ indicate that the estimated amplitude is quite stable with respect to variations in $r_{c}$, that is, that curves $A$ versus $r_{c}$ are fairly flat indeed. The observed bias in the determination of the amplitude is around $5-10 \%$, similar to that observed in simulations 1 and 2.

Finally, the compactness of the clusters embedded in white noise has the effect of increasing the number of spurious detections, especially for low detection thresholds. At the $5 \sigma$ level, however, the number of spurious sources is less than $5 \%$ of the true detections. Therefore, the scale-adaptive filter is well suited to detect and extract multiquadric profiles even in the less favorable case of more compact clusters and a background dominated by white noise.

\section{Comparison with Wavelets}

In order to compare the performance of the scale-adaptive filter with a wavelet-based analysis, we applied a Mexican Hat Wavelet and the difference of two Gaussians (eq. 17) to one of the simulations described in the previous section. Simulation 1 (section 4.1.1). was chosen for the sake of simplicity.

The scale-adaptive filter, by construction, directly gives the amplitude of the sources after filtering. On the contrary, the relation between the wavelet coefficients and the amplitude of the sources has to be calculated (or, alternatively, the wavelet has to be a priori re-normalized in order to directly give the amplitudes). Besides, the scale-adaptive filter for a multiquadric profile with a fixed scale is a function of the core radius $r_{c}$ (see eq. (5)) and therefore the variation of the scale of the filter relates directly with $r_{c}$. The parameter $r_{c}$ in eq. (5) corresponds exactly with the real $r_{c_{o}}$ of the clusters so that, due to condition (iii), the coefficient $w\left(r_{c}, \overrightarrow{0}\right)$ has a maximum when $r_{c}=r_{c_{o}}$. The relation between 
the width of the Mexican Hat or the difference of two Gaussians and $r_{c}$ is less clear and has to be analytically or numerically calculated. We suggest a procedure based on the calculation of the derivative of the wavelet coefficient with respect to the wavelet width.

For this test we filtered the same simulation (simulation 1) with three different 'filters': a scale-adaptive filter (see eq. (5)), a Mexican Hat Wavelet (eq. (15)) and a wavelet generated by the difference of two Gaussians (eq. (17)). For the scale-adaptive filter we repeated the same procedure described in section 4.1 .2 , that is, we tried to determine both the amplitude and the scale of the sources simultaneously. In the case of the wavelets, we performed an analogous analysis; instead of varying the parameter $r_{c}$ we varied the widths $R$. The relation between $R$ at the maximum and $r_{c}$ was analytically calculated for the Mexican Hat and the difference of two Gaussians. In a realistic case (with a priori unknown power spectrum of the noise) this relation must be calculated numerically. The relation between the coefficient at the waveleth width $R$ corresponding with the maximum and the amplitude $A$ was also analytically calculated. These calculations are not necessary in the case of scale-adaptive filter (they are included by construction).

The results of the test are showed in table 6 . The results of this test were compared taking into account five different aspects: sensitivity (that is, number of detections), reliability (proportion of spurious detections), gain, estimation of the amplitude and estimation of the core radius. Regarding the number of detections, there were no statistical differences between the three filters. The number of spurious detections, however, was significantly higher in the case of the difference of two Gaussians (4 spurious detections versus 1 in the case of the Mexican Hat and 0 in the case of the scale-adaptive filter). Comparing with table 3 we see that the number of spurious detections has dropped from 14 to 0 in the case of the scale-adaptive filter. This is due to the fact that we discard those 'source candidates' that are not detected in at least 5 filtered maps (as mentioned 
is section 4.1.2). Regarding gain, scale-adaptive filter works better than the two wavelets. In this simulation the number counts as a function of the amplitude of the sources is a constant, but in many realistic cases the number of sources grow quickly as they become fainter; in that case, any improvement in the gain of a filter will lead to a dramatic increase of the number of detections. Finally, the estimation of the amplitude and the radii of the clusters is similar in the three cases under study. The scale-adaptive filter seems to give slightly smaller errors in the determination of the core radii.

It is not difficult to explain the results of this test. The three considered filters are able to remove the large-scale background that dominates the image (the three of them drop to zero when $q \rightarrow 0$ ) and therefore give good results in the detection. However, the scale-adaptive filter gives better reliability in the detection and higher gains because its shape is better fitted to the profile of the sources. The Mexican Hat correlates well with Gaussian objects; if the source profile departs from the Gaussian this correlation is not perfect. Scale-adaptive filters are designed for a specific source profile; if different kind of objects are present in an image, a specific scale-adaptive filter can be constructed for any of them. Regarding the estimation of parameters, in this example the three filters work similarly because they have been used under the same philosophy. However, in the case of the Mexican Hat and the difference of two Gaussians it was necessary to find the relations between the coefficients and the amplitudes, and between the width of the wavelet at the maximum detection and $r_{c}$. In the simplistic case that has been considered these relations were straightforward because they could be calculated analytically. In a realistic case they should be calculated numerically, thus involving a maximization routine (with the consequent increase in processing time and complexity). Scale-adaptive filter does not suffer from this problem because the two mentioned relations are set equal to the identity by definition (conditions (i) and (iii)). 


\section{Conclusions}

In this paper we have used the concept of scale-adaptive filter and applied it to a multiquadric profile characterized by two parameters: the core radius $r_{c}$ and the the decay parameter $\lambda$, in order to obtain an unbiased estimation of the amplitude of the source. We have obtained explicit analytical formulas on Fourier space and simple analytical ones on real space for some source profiles and backgrounds. In particular, we focus on the interesting cases of microwave and X-ray emissions. A comparison with other standard filters is done. In particular, we remark that for $\gamma=n$ (e. g. $1 \mathrm{D}$ signal and $1 / f$ noise, 2D image and $P(q) \propto q^{-2}$ ) the scale-adaptive and matched filters coincide. Besides, a comparison with wavelet analysis techniques is performed. The goals of scale-adaptive filters and wavelets are not the same: while wavelets aim to fully describe structures in both space and frequency domains, scale-adaptive filters focus only in the problem of how to maximize the probability of detecting sources with a given profile with high reliability. Scale-adaptive filters adopt some features of the continuous wavelet transform, namely self-similarity and the ability to perform a sort of continuous multiresolution analysis. This analysis at multiple scales can be used to estimate the scale of sources and to improve the reliability of the detections. In this context, wavelets are more flexible and versatile, while filters are more powerful for a single yet important task.

We have simulated $2 \mathrm{D}$ clusters with $\lambda=1 / 2$, with different core radius $r_{c}$, embedded in

a background $P(q) \propto q^{-3}$ that can mimic microwave emission from the cluster plus intrinsic microwave and foreground emissions and noise. When the characteristic scale (the 'core radius' $r_{c}$ ) is known a priori we are able to recover a great number of sources without any significant error in their position and with errors in the determination of the amplitude of $\sim 10 \%$. About a $20 \%$ of spurious detections are also detected. This percentage of spurious sources could be reduced by introducing a multiple-scale analysis (i.e. imposing that the 
sources appear in a certain number of maps filtered with different scales). However, this would also reduce the number of true detections. The mean gain in this simulated case is 4.4, meaning that over a $5 \sigma$ detection threshold in the filtered maps we are able to detect sources that were only at $1.14 \sigma$ in the original map.

When the characteristic scale is not known a priori, as will happen in a realistic case, the size of the clusters can be estimated by filtering the map with several filters at different scales and looking for the maximum among the coefficients at the position of the sources. Moreover, the multiscale analysis can be used to reject spurious detections (that typically appear only in one or a few of the filtered maps) by imposing that the source 'candidates' must be present in several filtered maps. Unfortunately, the price of removing spurious detections is always to reduce the number of detections. We performed a simulation including clusters of different sizes and applied a multiscale analysis with ten filters of different scale. Only sources appearing in five or more filtered maps were considered as true detections. Under these conditions, we were able to recover $30 \%$ less sources than in our first simulation, but the number of spurious detections dropped to 0 . The position of the sources was again recovered with no significant error. The mean error in the determination of the amplitude is $<15 \%$. Additionally, we were able to determine the scale parameter of the detected clusters with mean errors of $\sim 0.15$ pixels.

We have also simulated $2 \mathrm{D}$ clusters with $\lambda=3 / 2$, with different core radius $r_{c}$, embedded in a background $P(q)=$ constant that can mimic X-ray emission from the cluster plus white noise. Once more, the multiscale analysis allows us to estimate the position, amplitude and scale of the sources with small errors. The results are slightly worse than in the microwave simulations because the X-ray clusters are more compact and because the background used in this simulation (white noise) is not the most favorable for the scale-adaptive filters. Scale-adaptive filters take advantage of the fact that in most cases 
the power spectrum of the noise has a maximum at a scale that is different from the scale of the sources. In the white noise case the power spectrum is constant at all scales. In other words, the scale-adaptive filter produced high gains in our microwave simulations because the background showed strong large-scale features that were removed efficiently by the scale-adaptive filters. In the white noise case, the gain is only $g=2.0$. In spite of that, the errors in the determination of the fundamental parameters remain small and the number of spurious detections over the $5 \sigma$ detection threshold is less than $5 \%$ of the number of true sources detected.

An additional point to address is 'source confusion', which is often a problem in deep images (X-ray or SZ maps) with a relatively large beam (with the exception of Chandra). Crowded fields are problematic for filtering as well as wavelet techniques. Source confusion can affect the performance of scale-adaptive filters in two ways. In the first place, the profile of two overlapped multiquadric profiles is, in general, not a multiquadric. Thus, the correlation between the filter and the overlapped sources will not be the ideal. In the second place, scale-adaptive filters work under the assumption that the power spectrum $P(q)$ of the data is not dominated by the sources we are trying to detect. In the case of one single source with no noise, for example, the scale-adaptive filter tends to the null filter. In that case, however, there is no point in filtering. Filtering is necessary in the low signal to noise regime, where sources do not dominate the image. Source confusion is not expected to be a problem in the future CMB Planck mission.

We conclude that the scale-adaptive filter is well suited to detect and extract multiquadric profiles embedded in generic homogeneous and isotropic backgrounds.

Scale-adaptive filters can be constructed for any source profile $\tau(x)$. If objects with different profiles are present, the image must be convolved with a set of scale-adaptive filters adapted for each kind of object. If the objects have different and not a priori known sizes 
the image can be convolved with a family of scale-adaptive filters of the same functional form while varying their scale. The number of scales probed with such family can be set by the user in order to sample the scale space with the desired precision. In this work spherical symmetry of the sources has been assumed, but this is not a fundamental requirement of the method. Scale-adaptive filters can be straightforwardly generalized in order to deal with non-symmetric profiles.

The filters presented in this work can be easily implemented and included in more general separation/detection methods. For example, the frequency dependence of the Sunyaev-Zel'dovich effect can be taken into account to perform the detection of clusters from multichannel microwave maps. Such method will be presented in a future work.

We thank Silvia Mateos, María Teresa Ceballos, Francisco Carrera and Patricio Vielva for useful suggestions and comments. DH acknowledges support from a Spanish MEC FPU fellowship. RBB acknowledges financial support from the PPARC in the form of a research grant. RBB thanks the Instituto de Física de Cantabria for its hospitality during a stay in 2001. We thank FEDER Project 1FD97-1769-c04-01, Spanish DGESIC Project PB98-0531-c02-01 and INTAS Project INTAS-OPEN-97-1192 for partial financial support. 


\section{REFERENCES}

Cayón, L., Sanz, J. L., Barreiro, R. B., Martínez-González, E., Vielva, P., Toffolatti, L., Silk, J., Diego, J. M. \& Argüeso, F., 2000, MNRAS, 315, 757

Damiani, F., Maggio, A., Micela, G. \& Sciortino, S., 1997, ApJ, 483, 350

Freeman, P.E., Kashyap, V., Rosner, R. \& Lamb, D.Q., accepted for publication in Ap. J. Supp. (v. 138 Jan. 2002).

Grebenev, S.A., Forman, W., Jones, C. \& Murray, S., 1995, ApJ, 445, 607.

Haykin, S., 1996, 'Adaptive filter theory', Prentice-Hall, New York.

Herranz, D., Gallegos, J., Sanz, J.L. \& Martínez-González, E., 2002, MNRAS in press.

Hobson, M.P., Jones, A.W., Lasenby, A.N. \& Bouchet, F.R., 1998, MNRAS, 300, 1.

Hobson, M.P., Barreiro, R.B., Toffolatti, L., Lasenby, A.N., Sanz, J.L., Jones, A.W., \& Bouchet, F.R. 1999, MNRAS, 306, 232.

Irwin, M.J., 1985, MNRAS, 214, 575.

Kawasaki, W., Shimasaku, K., Doi, M. \& Okamura, S., 1998, A\&A, 130, 567

Lazzati, D., Campana, S., Rosati, P., Panzera, M.R. \& Tagliaferri, G., 1999, ApJ, 524, 414.

Odgen, R.T., 'Essential wavelets for statistical applications and data analysis', 1997, Birkhäuser, Boston.

Postman, M., Lubin, L. M., Gunn, J. E., Oke, J. B., Hoessel, J. G., Schneider, D. P. \& Christensen, J. A., 1996, ApJ, 615, 111

Rosati, P., Della Ceca, R., Burg, R., Norman, C. \& Giacconi, R., 1995, ApJ, 445, L11. 
Sanz, J.L., Argüeso, F., Cayón, L., Martínez-González, E., Barreiro, R.B. \& Toffolatti, L., 1999a, MNRAS, 309, 672.

Sanz, J.L., Barreiro, R.B., Cayón, L., Martínez-González, E., Ruiz, G.A., Díaz, F.J., Argüeso, F., Silk, J. \& Toffolatti, L., 1999b, A\&AS, 140, 99.

Sanz, J. L., Herranz, D. \& Martínez-González, E., 2001, ApJ, 552, 484

Slezak, E., de Lapparent, V. \& Bijaoui, A., 1993, ApJ, 409, 517

Tegmark, M. \& Efstathiou, G. 1996, MNRAS, 281, 1927.

Tegmark, M. \& Oliveira-Costa, A. 1998, ApJ, 500, 83.

Valtchanov, I., Pierre, M. \& Gastaud, R., 2001, A\&A, accepted

Vielva, P., Martínez-González, E., Cayón, L., Diego, J. M., Sanz, J. L. \& Toffolatti, L., 2001a, MNRAS, 326, 181.

Vielva, P., Barreiro, R. B., Hobson, M., Martínez-González, E., Lasenby, A., Sanz, J. L. \& Toffolatti, L., 2001b, MNRAS, 328, 1.

Vikhlinin, A., Forman, W., Jones, C., \& Murray, S., 1995, ApJ, 451, 542.

Vikhlinin, A., et al., 1998, ApJ, 451, 542. 
Fig. 1.- Optimal scale-adaptive filters and matched filters (in Fourier space) associated to the $\beta=2 / 3$ profile for the cases $\tau(x)=\frac{1}{\left(1+(x / r c)^{2}\right)^{\lambda}}, \lambda=1 / 2$ (microwave) and $\lambda=3 / 2$ (X-ray) and a homogeneous and isotropic background with power spectrum $P(q) \propto p^{-\gamma}$. The two panels in the left of the figure show the optimal scale-adaptive filters for the cases $\gamma=0$ (solid line), $\gamma=1$ (dotted line), $\gamma=2$ (short-dashed line) and $\gamma=3$ (long-dashed line). There is a degeneracy for $\gamma=1$ and $\gamma=2$ in the two considered cases. The two panels in the right of the figure show the matched filters for the mentioned cases. For the matched filter and the case case $\lambda=1 / 2$ and $\gamma=0$ the modified profile $\tau(x)=N\left[\frac{1}{\sqrt{r_{c}^{2}+x^{2}}}-\frac{1}{\sqrt{r_{v}^{2}+x^{2}}}\right]$, $N=\frac{r_{c}}{1-r_{c} / r_{v}}$ has been used $\left(B=\frac{1}{4 \pi N \ln \left(\frac{r_{c}+r_{v}}{2 \sqrt{r_{c} r_{v}}}\right)}\right)$.

Fig. 2.- Simulation 1. Left panel shows the simulated 'clusters'. Central panel shows the data to be analyzed ('clusters' plus $q^{-3}$ noise). Right panel shows the coefficient map (after filtering). Only pixels above $3 \sigma$ have been plotted in the right panel.

Fig. 3.- Results of the detection (over a $3 \sigma$ threshold) in simulation 1 . The top panel shows the estimated versus the original amplitudes. Points $A=A_{o}$ are given by a dotted line. For the sake of clarity, error bars in the estimation of the amplitudes have not been represented; all of them have the same value that can be interpreted as the dispersion $\sigma$ of the filtered map. For this simulation, $\sigma=0.064$. The theoretical value of $\sigma$ can be calculated using eq. (9). The bottom panel shows the gain of the detected sources against the original amplitude. The mean gain is given by the horizontal dotted line.

Fig. 4.- Simulation 2. Left panel shows the simulated 'clusters'. Central panel shows the data to be analyzed ('clusters' plus $q^{-3}$ noise). Right panel shows the coefficient map (after filtering with an scale-adaptive filter of $\left.r_{c}=1.50\right)$.

Fig. 5.- Results of the detection (over a $3 \sigma$ threshold) in simulation 2. The top panel shows the estimated versus the original original core radii. The dotted line corresponds to 
$r_{c}=r_{c_{o}}$. The bottom panel shows the estimated versus the original amplitudes. For the sake of clarity, error bars in the estimation of the amplitudes have not been represented; all of them have the same value that can be interpreted as the dispersion $\sigma$ of the filtered map. For this simulation, $\sigma=0.071$. The theoretical value of $\sigma$ can be calculated using eq. (9). Points $A=A_{o}$ are given by a dotted line.

Fig. 6.- Simulation 3. Left panel shows the simulated 'clusters'. Central panel shows the data to be analyzed ('clusters' plus white noise). Right panel shows the coefficient map (after filtering with an scale-adaptive filter of $r_{c}=3.0$ ).

Fig. 7.- Results of the detection (over a $3 \sigma$ threshold) in simulation 3. The top panel shows the estimated versus the original original core radii. The dotted line corresponds to $r_{c}=r_{c_{o}}$. The bottom panel shows the estimated versus the original amplitudes. For the sake of clarity, error bars in the estimation of the amplitudes have not been represented; all of them have the same value that can be interpreted as the dispersion $\sigma$ of the filtered map. For this simulation, $\sigma=0.15$. The theoretical value of $\sigma$ can be calculated using eq. (9). Points $A=A_{o}$ are given by a dotted line. 


\begin{tabular}{cccc}
\hline \hline$\lambda$ & $\gamma$ & $\tilde{\Psi}_{o}(q)$ & $\Psi_{o}(x)$ \\
\hline $1 / 2$ & 0 & $\frac{2}{\pi} e^{-y}$ & $\frac{2}{\pi r_{c}^{2}} \frac{1}{\left(1+x^{2}\right)^{3 / 2}}$ \\
$1 / 2$ & 1 & $\frac{2}{\pi} y e^{-y}$ & $\frac{2}{\pi r_{c}^{2}} \frac{2-x^{2}}{\left(1+x^{2}\right)^{5 / 2}}$ \\
$1 / 2$ & 2 & $\frac{2}{\pi} y e^{-y}$ & $\frac{2}{\pi r_{c}^{2}} \frac{2-x^{2}}{\left(1+x^{2}\right)^{5 / 2}}$ \\
$1 / 2$ & 3 & $\frac{2}{\pi}(3-y) y^{2} e^{-y}$ & $\frac{4}{\pi r_{c}^{2}} \frac{-12 x^{4}+21 x^{2}-2}{\left(1+x^{2}\right)^{9 / 2}}$ \\
$3 / 2$ & 0 & $\frac{2}{\pi}(2 y-1) e^{-y}$ & $\frac{6}{\pi r_{c}^{2}} \frac{1-x^{2}}{\left(1+x^{2}\right)^{5 / 2}}$ \\
$3 / 2$ & 1 & $\frac{4}{3 \pi} y^{2} e^{-y}$ & $\frac{4}{3 \pi r_{c}^{2}} \frac{2-3 x^{2}}{\left(1+x^{2}\right)^{7 / 2}}$ \\
$3 / 2$ & 2 & $\frac{4}{3 \pi} y^{2} e^{-y}$ & $\frac{4}{3 \pi r_{c}^{2}} \frac{2-3 x^{2}}{\left(1+x^{2}\right)^{7 / 2}}$ \\
$3 / 2$ & 3 & $\frac{4}{15 \pi}(5-y) y^{3} e^{-y}$ & $\frac{4}{3 \pi r_{c}^{2}} \frac{9 x^{6}-84 x^{4}+88 x^{2}-2}{\left(1+x^{2}\right)^{11 / 2}}$ \\
\hline
\end{tabular}

Table 1: Optimal scale-adaptive filters associated to the $\beta=2 / 3$ profile for the cases $\tau(r)=$ $\frac{1}{\left(1+(r / r c)^{2}\right)^{\lambda}}, \lambda=1 / 2$ (microwave) and $\lambda=3 / 2$ (X-ray).

Note. - Col. (1): $\lambda$. Col. (2): Background (noise) exponent $\gamma$. Col (3): Filter in Fourier space $\left(y=q r_{c}\right)$. Col. (4): Filter in real space $\left(x=r / r_{c}\right)$. 


\begin{tabular}{|c|c|c|c|}
\hline$\lambda$ & $\gamma$ & $\tilde{\Psi}_{o}(q)$ & $\Psi_{o}(x)$ \\
\hline $1 / 2$ & 0 & $B \frac{e^{-q r_{c}}-e^{-q r_{v}}}{q}$ & $\frac{B}{N} \tau(x)$ \\
\hline $1 / 2$ & 1 & $\frac{1}{\pi} e^{-q r_{c}}$ & $\frac{1}{\pi r_{c}^{2}} \frac{1}{\left(1+x^{2}\right)^{3 / 2}}$ \\
\hline $1 / 2$ & 2 & $\frac{2}{\pi}\left(q r_{c}\right) e^{-q r_{c}}$ & $\frac{2}{\pi r_{c}^{2}} \frac{2-x^{2}}{\left(1+x^{2}\right)^{5 / 2}}$ \\
\hline $1 / 2$ & 3 & $\frac{2}{\pi}\left(q r_{c}\right)^{2} e^{-q r_{c}}$ & $\frac{2}{\pi r_{c}^{2}} \frac{2-3 x^{2}}{\left(1+x^{2}\right)^{7 / 2}}$ \\
\hline $3 / 2$ & 0 & $\frac{2}{\pi} e^{-q r_{c}}$ & $\frac{2}{\pi r_{c}^{2}} \frac{1}{\left(1+x^{2}\right)^{3 / 2}}$ \\
\hline $3 / 2$ & 1 & $\frac{2}{\pi}\left(q r_{c}\right) e^{-q r_{c}}$ & $\frac{2}{\pi r_{c}^{2}} \frac{2-x^{2}}{\left(1+x^{2}\right)^{3 / 2}}$ \\
\hline $3 / 2$ & 2 & $\frac{4}{3 \pi}\left(q r_{c}\right)^{2} e^{-q r_{c}}$ & $\frac{4}{3 \pi r_{c}^{2}} \frac{2-3 x^{2}}{\left(1+x^{2}\right)^{3 / 2}}$ \\
\hline $3 / 2$ & 3 & $\frac{2}{3 \pi}\left(q r_{c}\right)^{3} e^{-q r_{c}}$ & $\frac{2}{\pi r_{c}^{2}} \frac{3 x^{4}-24 x^{2}+8}{\left(1+x^{2}\right)^{3 / 2}}$ \\
\hline
\end{tabular}

Table 2: Matched filters associated to the $\beta=2 / 3$ profile for the cases $\tau(r)=\frac{1}{\left(1+(r / r c)^{2}\right)^{\lambda}}$, $\lambda=1 / 2$ (microwave) and $\lambda=3 / 2$ (X-ray). For the case $\lambda=1 / 2$ and $\gamma=0$ the modified profile $\tau(r)=N\left[\frac{1}{\sqrt{r_{c}^{2}+r^{2}}}-\frac{1}{\sqrt{r_{v}^{2}+r^{2}}}\right], N=\frac{r_{c}}{1-r_{c} / r_{v}}$ has been used $\left(B=\frac{1}{4 \pi N \ln \left(\frac{r_{c}+r_{v}}{2 \sqrt{r_{c} r_{v}}}\right)}\right)$.

Note. - Col. (1): $\lambda$. Col. (2): Background (noise) exponent $\gamma$. Col (3): Filter in Fourier space $\left(y=q r_{c}\right)$. Col. (4): Filter in real space $\left(x=r / r_{c}\right)$. 


\begin{tabular}{ccccccc}
\hline \hline$\sigma$ & detected & spurious & mean offset & $\bar{b}_{A}$ & $\bar{e}_{A}$ & $\bar{g}$ \\
\hline 3.0 & 80 & 14 & 0.0 & 7.7 & 10.7 & 4.4 \\
4.0 & 71 & 17 & 0.0 & 6.6 & 9.5 & 4.4 \\
5.0 & 59 & 12 & 0.0 & 7.2 & 9.7 & 4.4 \\
\hline
\end{tabular}

Table 3: Detections in simulation 1. The number of simulated sources is 100.

Note. - Col. (1): $\sigma$ detection level. Col. (2): Number of true detections above the $\sigma$ threshold. Col (3): Number of spurious sources. Col. (4): Mean offset in the position of the source (pixels). Col. (5): Mean relative bias in the determination of the amplitude (\%), defined as the average of $100 \times \frac{A-A_{o}}{A_{o}}$. Col. (6): Mean relative error in the determination of the amplitude (\%), defined as the average of $100 \times \frac{\left|A-A_{o}\right|}{A_{o}}$. Col. (7): Mean gain (as defined in eq. (14)) 


\begin{tabular}{ccccccccc}
\hline \hline$\sigma$ & detected & spurious & mean offset & $\bar{b}_{A}$ & $\bar{e}_{A}$ & $\bar{b}_{r_{c}}$ & $\bar{e}_{r_{c}}$ & $\bar{g}$ \\
\hline 3.0 & 57 & 0 & 0.0 & 11.5 & 14.0 & -0.12 & 0.14 & 3.7 \\
4.0 & 49 & 0 & 0.0 & 7.3 & 10.4 & -0.13 & 0.14 & 3.5 \\
5.0 & 38 & 0 & 0.0 & 6.1 & 8.5 & -0.12 & 0.13 & 3.5 \\
\hline
\end{tabular}

Table 4: Detections in simulation 2. The number of simulated sources is 100.

Note. - Col. (1): $\sigma$ detection level. Col. (2): Number of true detections above the $\sigma$ threshold. Col (3): Number of spurious sources. Col. (4): Mean offset in the position of the source (pixels). Col. (5): Mean relative bias in the determination of the amplitude (\%), defined as the average of $100 \times \frac{A_{i}-A_{o_{i}}}{A_{o_{i}}}$. Col. (6): Mean relative error in the determination of the amplitude (\%), defined as the average of $100 \times \frac{\left|A_{i}-A_{o_{i}}\right|}{A_{o_{i}}}$. Col. (7): Mean bias in the determination of $r_{c}$ (in pixel units), defined as the average of $\frac{r_{c_{i}}-r_{c_{o_{i}}}}{r_{c_{o_{i}}}}$. Col. (8): Mean absolute error in the determination of $r_{c}$ (pin pixel units), defined as the average of $\frac{\left|r_{c_{i}}-r_{c_{o_{i}}}\right|}{r_{c_{o_{i}}}}$. Col. (9): Mean gain (as defined in eq. (14)) 


\begin{tabular}{ccccccccc}
\hline \hline$\sigma$ & detected & spurious & mean offset & $\bar{b}_{A}$ & $\bar{e}_{A}$ & $\bar{b}_{r_{c}}$ & $\bar{e}_{r_{c}}$ & $\bar{g}$ \\
\hline 3.0 & 73 & 22 & 0.4 & 10.1 & 17.8 & 0.06 & 0.40 & 2.0 \\
4.0 & 63 & 10 & 0.3 & 6.2 & 14.9 & 0.03 & 0.39 & 2.0 \\
5.0 & 48 & 2 & 0.2 & 6.9 & 12.6 & 0.05 & 0.36 & 2.0 \\
\hline
\end{tabular}

Table 5: Detections in simulation 3. The number of simulated sources is 100.

Note. - Col. (1): $\sigma$ detection level. Col. (2): Number of true detections above the $\sigma$ threshold. Col (3): Number of spurious sources. Col. (4): Mean offset in the position of the source (pixels). Col. (5): Mean relative bias in the determination of the amplitude (\%), defined as the average of $100 \times \frac{A_{i}-A_{o_{i}}}{A_{o_{i}}}$. Col. (6): Mean relative error in the determination of the amplitude (\%), defined as the average of $100 \times \frac{\left|A_{i}-A_{o_{i}}\right|}{A_{o_{i}}}$. Col. (7): Mean bias in the determination of $r_{c}$ (in pixel units), defined as the average of $\frac{r_{c_{i}}-r_{c_{o_{i}}}}{r_{c_{o_{i}}}}$. Col. (8): Mean absolute error in the determination of $r_{c}$ (in pixel units), defined as the average of $\frac{\left|r_{c_{i}}-r_{c_{o_{i}}}\right|}{r_{c_{o_{i}}}}$. Col. (9): Mean gain (as defined in eq. (14)) 


\begin{tabular}{cccccccc}
\hline \hline Filter & detected & spurious & $\bar{g}$ & $\bar{b}_{A}$ & $\bar{e}_{A}$ & $\bar{b}_{r_{c}}$ & $\bar{e}_{r_{c}}$ \\
\hline SAF & 87 & 0 & 4.4 & -11.1 & 13.4 & -0.08 & 0.08 \\
MHW & 89 & 1 & 4.2 & -10.1 & 13.4 & -0.09 & 0.13 \\
D2G & 88 & 4 & 4.2 & -12.2 & 13.5 & -0.08 & 0.11 \\
\hline
\end{tabular}

Table 6: Comparison of the performances of the scale-adaptive filter and two wavelets. The analysis was performed over detections at the $3 \sigma$ detection level using Simulation 1.

Note. - Col. (1): Filter type (SAF=scale-adaptive filter, MHW=Mexican Hat Wavelet, D2G=Difference of two Gaussians). Col. (2): Number of true detections above the $\sigma$ threshold. Col (3): Number of spurious sources. Col. (4): Mean gain (as defined in eq. (14)) Col. (5): Mean relative bias in the determination of the amplitude (\%), defined as the average of $100 \times \frac{A-A_{o}}{A_{o}}$. Col. (6): Mean relative error in the determination of the amplitude (\%), defined as the average of $100 \times \frac{\left|A-A_{o}\right|}{A_{o}}$. Col. (7): Mean bias in the determination of $r_{c}$ (in pixel units), defined as the average of $\frac{r_{c_{i}}-r_{c_{o_{i}}}}{r_{c_{o_{i}}}}$. Col. (8): Mean absolute error in the determination of $r_{c}$ (in pixel units), defined as the average of $\frac{\left|r_{c_{i}}-r_{c_{o_{i}}}\right|}{r_{c_{o_{i}}}}$. 

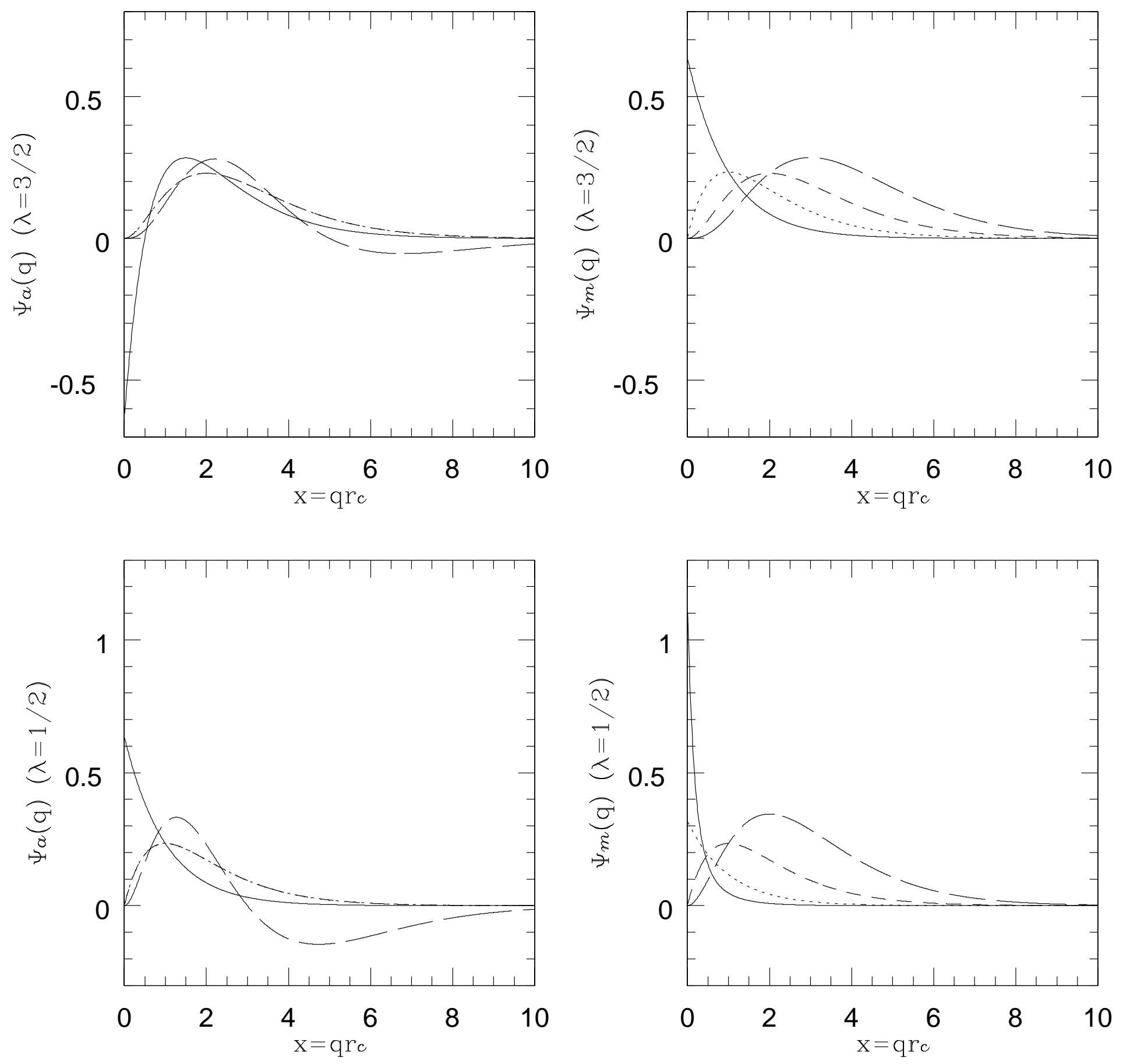


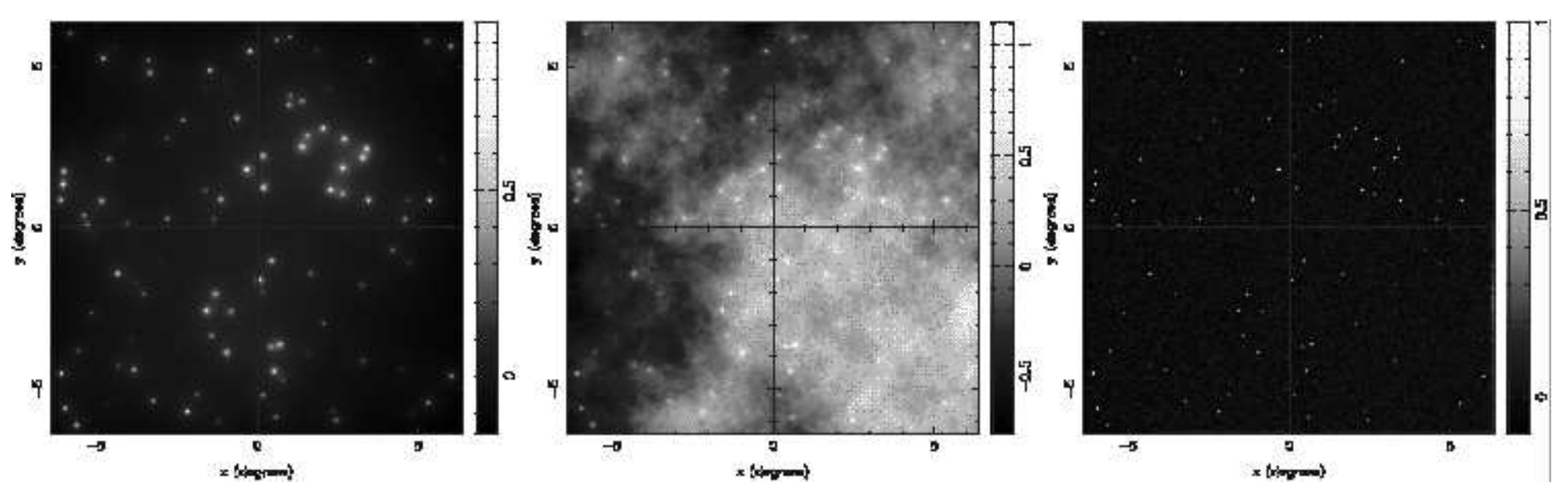



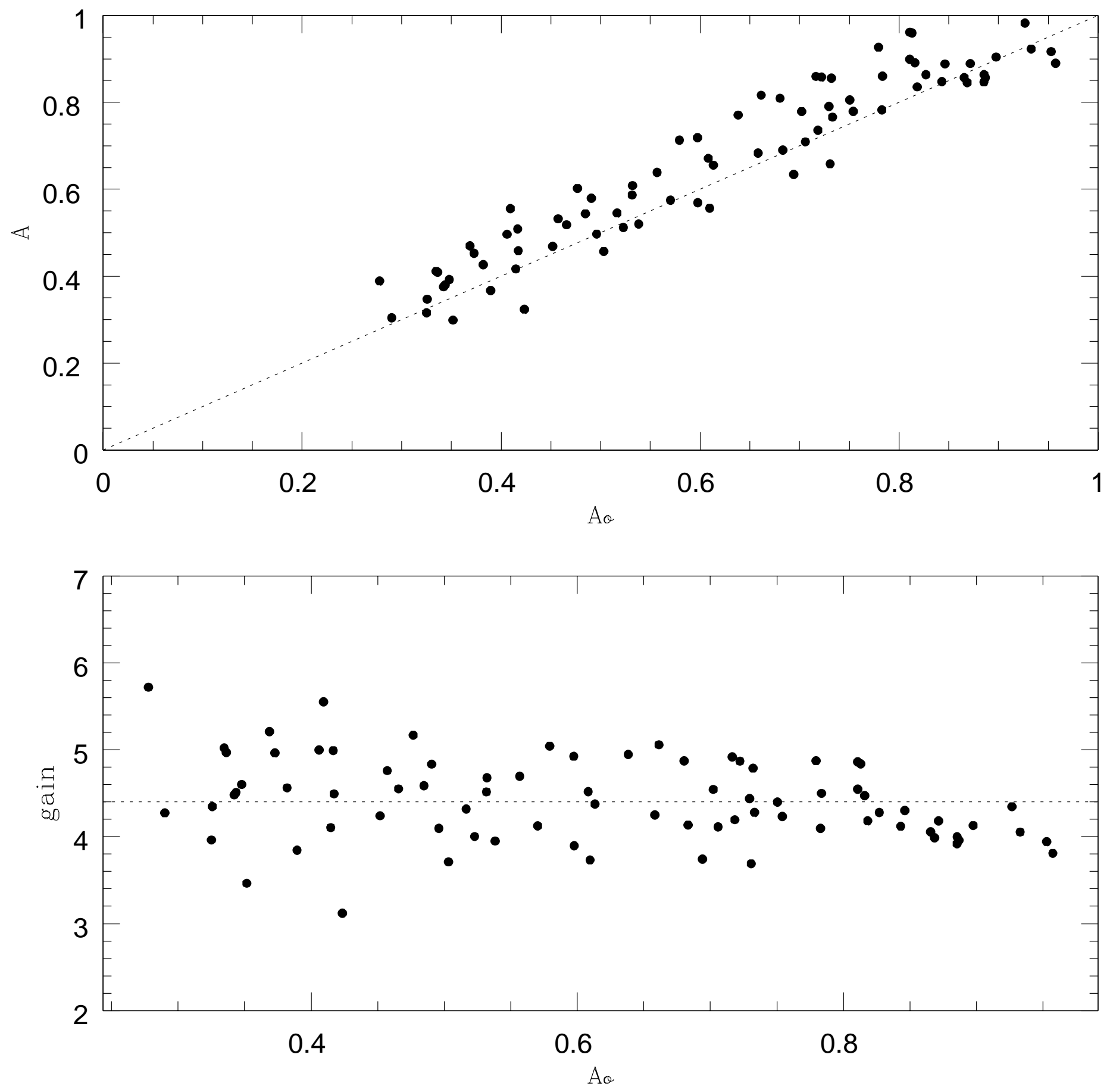

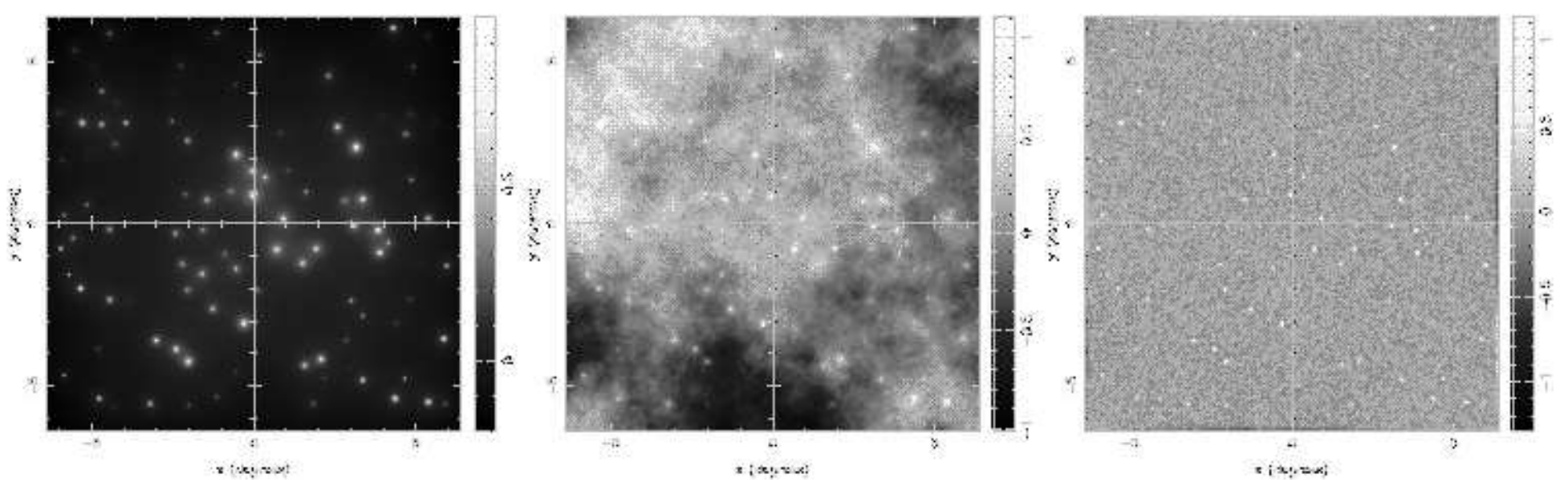

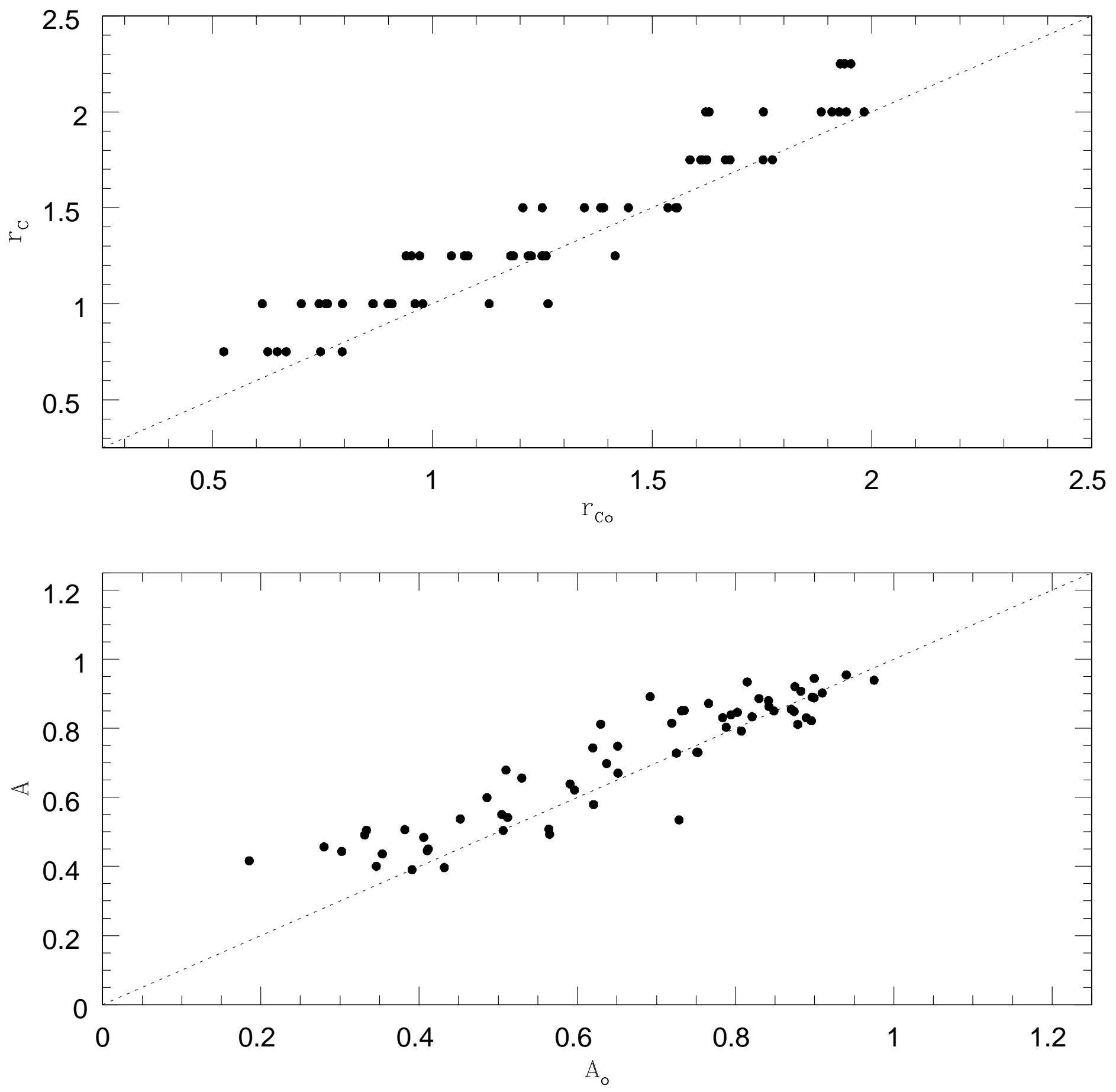

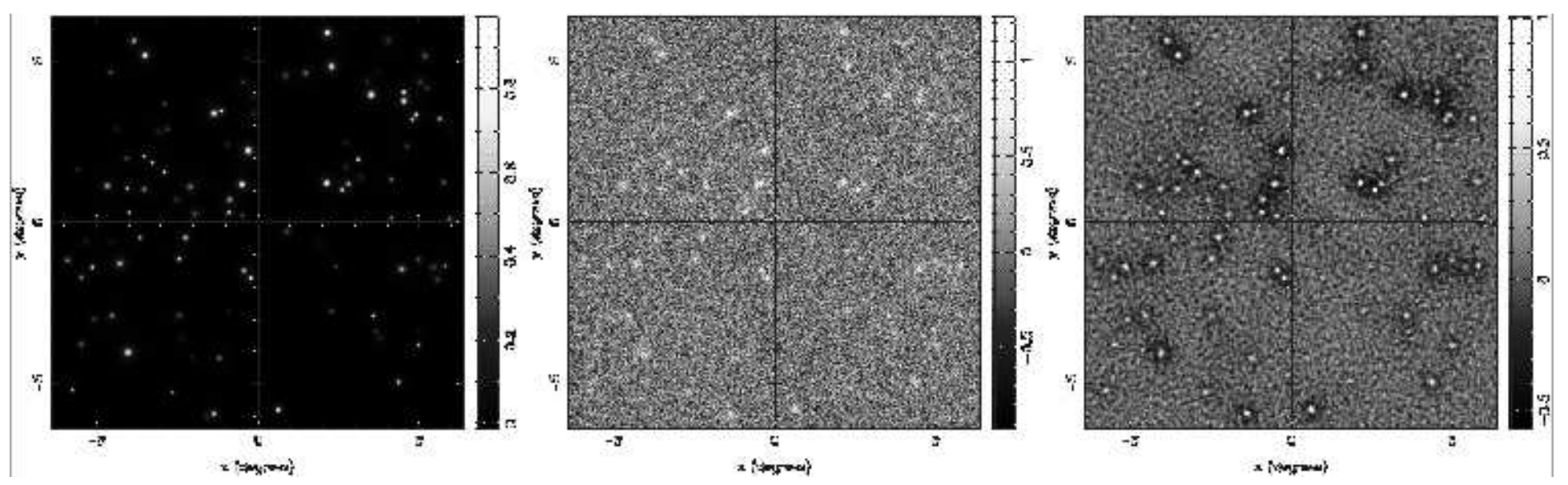

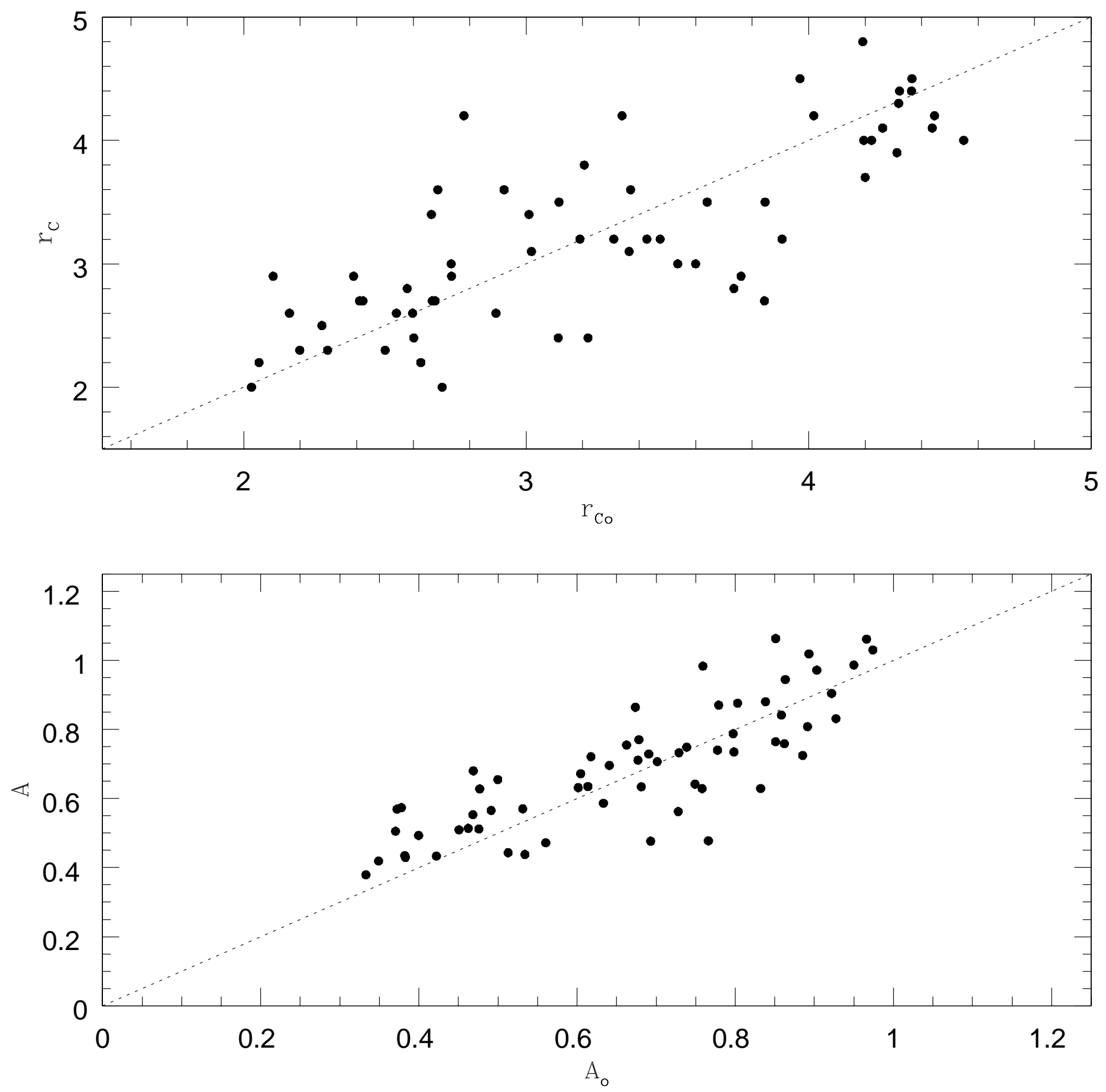